\title{
604 COMBINING A NOVEL DUAL RAF/MEK INHIBITOR WITH IMMUNOMODULATION TO PROMOTE AN ANTI-TUMOR RESPONSE
}

Lauren Dong*, Hyejin Choi, Sadna Budhu, Isabell Schulze, Nezar Mehanna, Neal Rosen, Taha Merghoub, Jedd Wolchok. Memorial Sloan Kettering Cancer Center, New York, NY, USA

Background The therapeutic scope of MEK inhibitors (MEKis) is currently limited to use in BRAF mutant melanoma. Therefore, we aim to develop new strategies to extend their usage to MEKi resistant RAS mutant cancers, which represent an unmet clinical need. In Ras mutant murine lung cancers, CH5126766 (CKI27) is novel due to its ability to inhibit both RAF and MEK, preventing the rebound of p-ERK that normally results from the relief of negative feedback in the MAPK/ERK pathway. However, CKI27 is also capable of inhibiting $\mathrm{T}$ cell functions because the MAPK/ERK pathway is activated downstream of $\mathrm{T}$ cell receptor signaling. We aim to balance the positive and negative immunomodulatory effects of MEKis for optimal combination with immunotherapy.

Methods To evaluate the effects of CKI27 on tumor cells and $\mathrm{T}$ cells in vitro, we performed flow cytometry, cytokine analysis, and functional co-culture assays. Lewis lung carcinoma (LLC) tumor bearing mice were treated either with CKI27 combined with co-stimulatory agonist antibody targeting GITR and checkpoint blockade antibody targeting CTLA-4 or the appropriate controls to determine efficacy and changes in the tumor microenvironment.

Results We observed that CKI27 increased MHC expression on tumor cells and $\mathrm{T}$ cell mediated killing. Yet, CKI27 also decreased $\mathrm{T}$ cell proliferation, activation, and cytolytic activity. Implementing a break for $\mathrm{T}$ cells to recover with intermittent dosing of CKI27 partially relieved these inhibitory effects. Further combination with agonist antibodies anti-OX40 and GITR completely alleviated these $\mathrm{T}$ cell toxicities and increased combination efficacy with checkpoint blockade antibody anti-CTLA-4.

Conclusions Understanding the immunomodulatory effects of combining CKI27 with immunotherapy will elucidate the mechanism behind their increased efficacy. This will allow us to make more informed decisions in dosing regimens, overcoming resistance, and generating long-term immune responses in current and future clinical trials treating patients with RAS mutant cancers.

http://dx.doi.org/10.1136/jitc-2021-SITC2021.604 